\title{
Flow characteristics and noise reduction effects of air cleaners of automobile intake systems with built-in resonators with space efficiency
}

\author{
Hoseop Song and Haengmuk Cho* \\ Dept. of Mechanical Engineering, Kongju National University, Republic of Korea \\ *Corresponding Author: haengmukcho@hanmail.net
}

Submitted: $27 / 06 / 2019$

Revised: $\quad 02 / 09 / 2019$

Accepted: 19/02/2020

\begin{abstract}
In an automobile intake system, Helmholtz resonator is often used to reduce intake noise. But there is a disadvantage in the engine room. Resonator brings low engine room space efficiency, because resonator needs some. In this paper, we propose air cleaner with built-in resonator for a limited space of air cleaner and analyze the pressure drop effect and the transmission loss performance of the resonator through flow analysis. The air cleaner with a built-in resonator had almost no difference in pressure drop with the conventional air cleaner. Some vortex in conventional air cleaner is removed, and also, the built-in resonator has noise reduction effect at $160 \mathrm{~Hz}$ at $61 \mathrm{~dB}$ such as same resonator.
\end{abstract}

Keywords: Intake system; Intake noise; Resonator; Fluids analysis; Transmission loss.

\section{INTRODUCTION}

In an automobile, an intake system refers to an overall system delivering external air to the engine. The schematic of intake system is in figure 1, and it consists of the snorkel that sucks air from the outside, the air cleaner that purifies the air, the duct, the throttle valve for controlling air flow rate, and the manifold that distributes air to each cylinder. Among these components, the air cleaner takes up the largest volume, comprised of an upper part and a lower part, inside which a filter is attached to purify the air.

In this intake system, intake noise is generated. Intake noise is low and blunt noise under $600 \mathrm{~Hz}$. It consists of intake discharge noise, surface radiation sound, and pipe radiation sound, and the main cause is the pressure pulse of air current, caused by engine motion, and resonance effect inside the intake component. This is takes up 30\% of automobile noise, and studies have been carried out to suppress it (Tetsushi Suzuki et al., 1990).

After Brands propose Helmholtz resonator for intake system (M. C. Brands et al. 1979), many studies used the Helmholtz resonator to suppress intake noise (M. S. Kim et al., 2006 \& S.K. Tang., 2005 \& J. Lee, 2009 \& S. H. Kim, 2014), and recently, Kitahara et al. studied the effect of using a porous duct instead of a plastic duct to reduce some noise (S. Kitahara et al, 2005). However, the Helmholtz resonator targets a specific frequency band to bring high noise reduction effect in that frequency band, and the acoustic duct has the advantages and disadvantages of lowering the resonance compared to the overall frequency in the entire frequency band (M. S. Kim et al., 2006 \& J. Lee, 2009 \& H. Y. Song, 2008 ). However, since the resonator is installed, the efficiency of the engine room space of the vehicle is deteriorated and the workability is deteriorated. Thus, there is a need for a more efficient design (S. Allam, 2015). The noise reduction method using the resonator is a frequently used method in the intake system (U. Patil et al., 2019). 


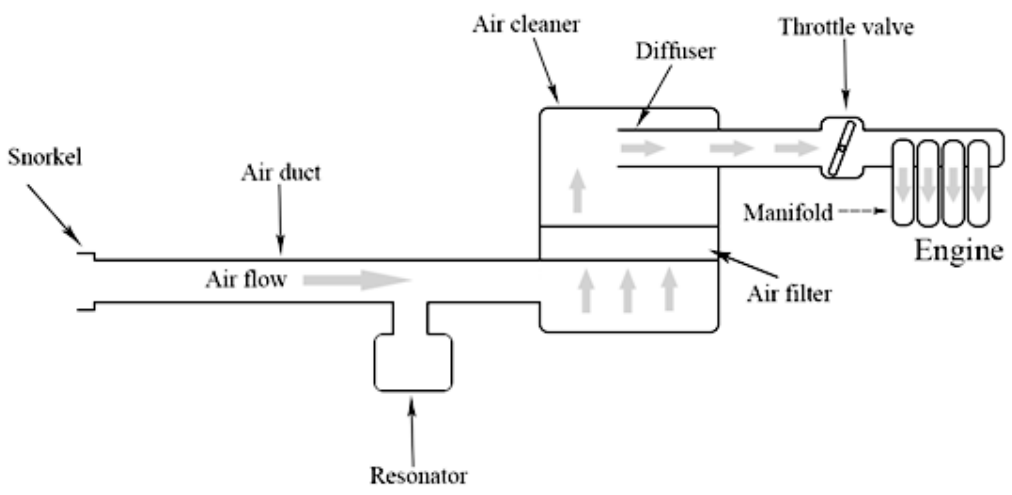

Figure 1. Schematic of intake system of automobile.

B.M. Yang et al. get a result about intake system flow pattern and noise reduction effect by double resonator system (B.M. Yang et al., 2016).

Vishal Vaidya et al. tested the effect on the resonator in intake system, and they conclude resonator has significant effect on the transmission loss and reduce transmission loss at overall sound pressure level (Vishal Vaidya et al., 2014).

In this paper, an air cleaner model with a built-in resonator that reduces noise and increases space efficiency by attaching a resonator in a specific space of the air cleaner used in automobiles. It also aims to analyze noise reduction performance and the pressure drop effect caused by the resonator using the transmission loss method and CFD.

\section{METHODOLOGY}

\section{Helmholtz Resonator}

The Helmholtz resonator is a device based on the resonance effect with a combination of the cavity, cross-section of the neck and the length of neck which is an effective noise reduction device in low frequency bands. Helmholtz resonator is shown in Figure 2. Fundamentally comprised of an isolated cavity and a neck connected to the outside, the air inside the cavity acts as a spring to cause resonance, and upon resonance, air in the neck part goes in and out during resonance and is converted into thermal energy by friction with the wall. Equation 1 is an equation for the calculation of the resonance frequency $\left(f_{r}\right)$ of the Helmholtz resonator.

$$
f_{r}=\frac{c_{0}}{2 \pi} \sqrt{\frac{A_{n}}{l_{n} V_{c}}}
$$

Here, $c_{0}$ denotes sonic speed, $A_{n}$ denotes the cross-section of the neck, $1_{n}$ denotes the length of the neck, and $V_{c}$ denotes the volume of the cavity. Based on this equation, the volume of the cavity or the length of the neck increases, or as the cross-section of the neck shrinks, the resonance frequency decreases (J. E. Kim, 2015). However, in most engine rooms, lowering the frequency band by increasing the volume in order to reduce the noise in the low frequency band is limited by the space limitation in the engine room. 


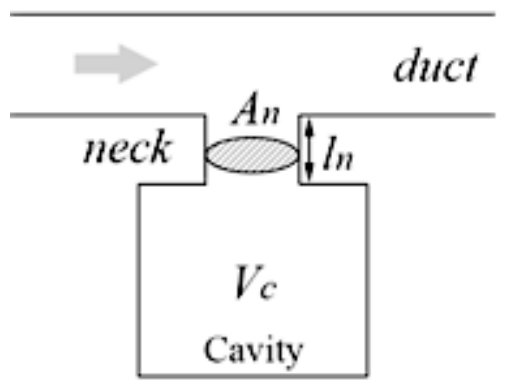

Figure 2. Schematic of Helmholtz resonator.

\section{Transmission Loss}

The transmission loss is defined as the ratio of the incident sound power of the silencer to the acoustic power $\left(W_{t}\right)$ and the ratio of the sound output of the silencer $\left(W_{i}\right)$. Equation 2 shows a basic expression for transmission loss.

$$
\mathrm{TL}=10 \log \frac{W_{t}}{W_{i}}
$$

The transmission loss value shows how much sound wave information has passed through, and the higher the value, the more the noise not passed. Therefore, the TL value is used as a measure to evaluate the performance of the silencer. In this paper, the performance of the resonator will be confirmed through this index.

\section{ANALYSIS OF AIR CLEANER}

\section{Automobile Air Cleaner}

In order to use the air cleaner for the intake system used in the actual vehicle as an analytical model, a simplified model was prepared except for unnecessary parts. It consists of 3 parts, including the lower part through which air enters, the filter part where the filter is located, and the upper part, where the diffuser and the $1 / 4$ wavelength pipe are located. Figure 3 is a model showing the internal flow path of a simplified air cleaner, where the 3 parts can be seen separately. Also, Figure 4 shows the upper part, where the diffuser and the 1/4 wavelength pipe are located.

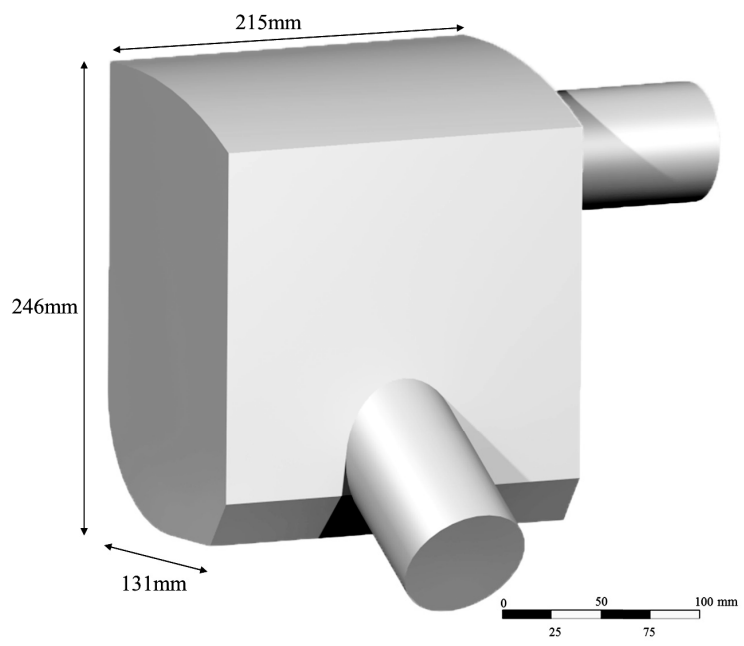

Figure 3. Simplified model of air cleaner for automobile. 


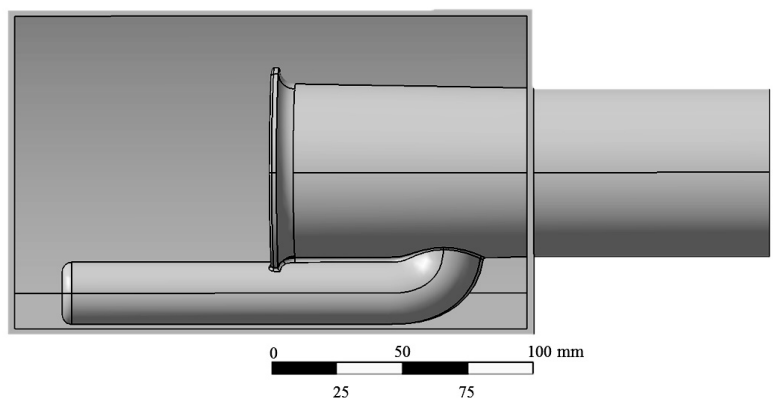

Figure 4. Diffuser and side branch resonator.

\section{Analyzing Conditions of Air Cleaner}

For the installation of the new resonator, a flow analysis was carried out to find out the part of the flow in the existing air cleaner model and to analyze the internal flow. The measured values at $3500 \mathrm{rpm}$, which were measured from real car condition, were used to assume the driving conditions, and the values are shown in Table 1. The mesh has about 1.44 million elements, and tetra model was used, and the prism model was used for inflation considering the flow with the wall. In order to reproduce the turbulent flow assuming the flow in the pipe to be incompressible full turbulence, the most stable model, k- $\varepsilon$ Realizable model, was used to perform the analysis.

In order to describe the filter part of the air cleaner, Porous setting using Fluent was used, and loss values were applied using Darcey's Law. The viscous resistance equation is shown in Equation 3, the inertial resistance equation is shown in Equation 4, and the calculated values are shown in Table 2.

$$
\begin{gathered}
\frac{1}{K_{\text {perm }}}=\frac{150}{D_{p}{ }^{2}} \frac{(1-\epsilon)^{2}}{\epsilon^{3}} \\
K_{\text {loss }}=\frac{3.5}{D_{p}} \frac{(1-\epsilon)}{\epsilon^{3}}
\end{gathered}
$$

$K_{\text {perm }}$ is the viscous resistance, $K_{\text {loss }}$ is inertial resistance, $D_{p}$ is the depth of the filter, and $\epsilon$ is the porosity of the filter.

Table 1. Boundary conditions.

\begin{tabular}{|c|c|}
\hline Condition & Value \\
\hline Mass flow rate & $171 \mathrm{~kg} / \mathrm{h}$ \\
\hline Inlet pressure & $-1322 \mathrm{~Pa}$ \\
\hline Outlet Pressure & Atmosphere pressure \\
\hline Air Density & $1.225 \mathrm{~kg} / \mathrm{m}^{3}$ \\
\hline Air Viscosity & $1.7894 \mathrm{~kg} / \mathrm{m} \cdot \mathrm{s}$ \\
\hline
\end{tabular}

Table 2. Porosity media conditions.

\begin{tabular}{|c|c|}
\hline Condition & Value \\
\hline Filter porosity & 0.85 \\
\hline Viscous Resistance & $2487.831 / \mathrm{m}^{2}$ \\
\hline Inertial Resistance & $18.18881 / \mathrm{m}$ \\
\hline
\end{tabular}




\section{Fluid Characteristic Analysis of Air Cleaner}

Analysis of the fluid characteristics of the air cleaner showed that most of the air entered through the lower part, passed through the filter, sucked from the diffuser in the upper part, and exited the air cleaner. (a) of Figure 5 is the internal streamline, and most of the flow was absorbed straight into the diffuser, but some flow was unable to enter the diffuser and instead remained in the lower part of the air cleaner like figure (b). And that air makes an unnecessary vortex at the bottom of the air cleaner. Also, as shown in (c) of Figure 5, the pressure was high where the air hits first the wall and where it is blocked by the $1 / 4$ wavelength pipe of the upper part. In the lower part of the diffuser, there were parts where pressure decreased drastically, which caused by a rapid decrease in pressure as air is suddenly sucked into the diffuser, causing a high velocity.

Therefore, placing the newly installed resonator in the lower part of the air cleaner where the vortex is formed, where the flow path is least affected, would be most efficient. Thus, it is aimed to install a new wall in that area and make a separate space to serve as the cavity for the resonator.

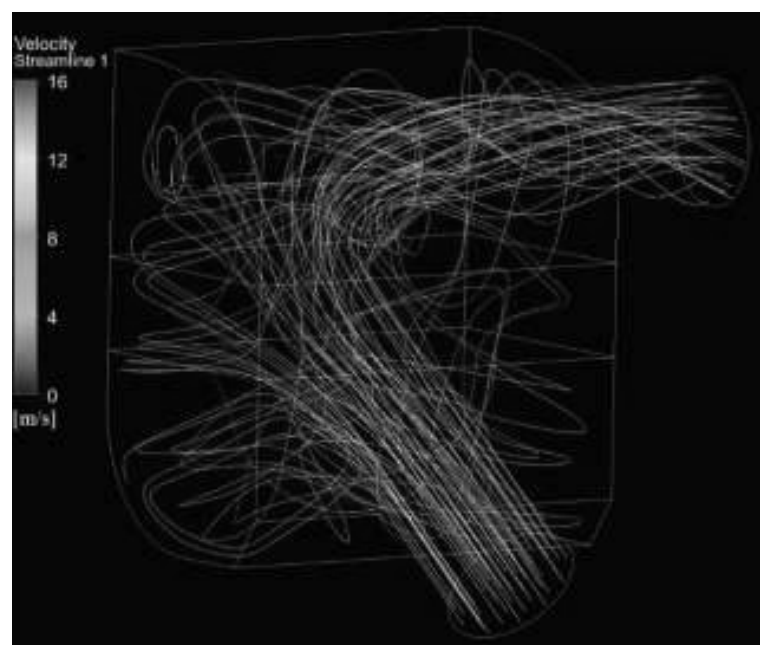

(a) Streamline in air cleaner

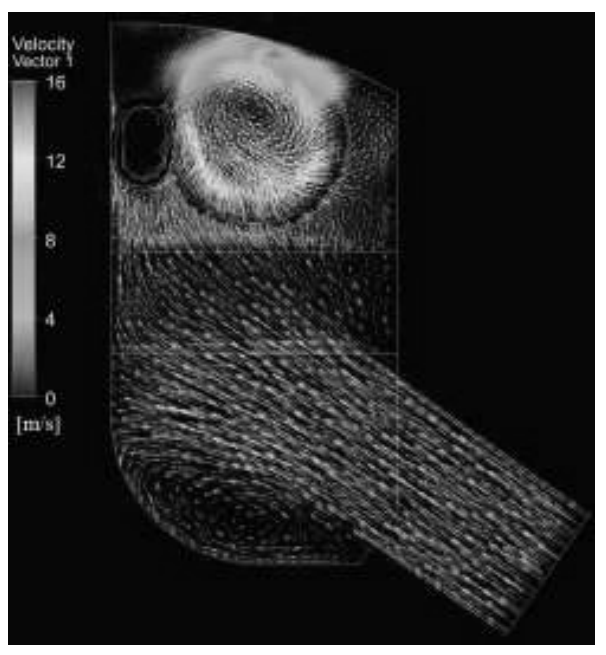

(b) Vector in cross section of air cleaner

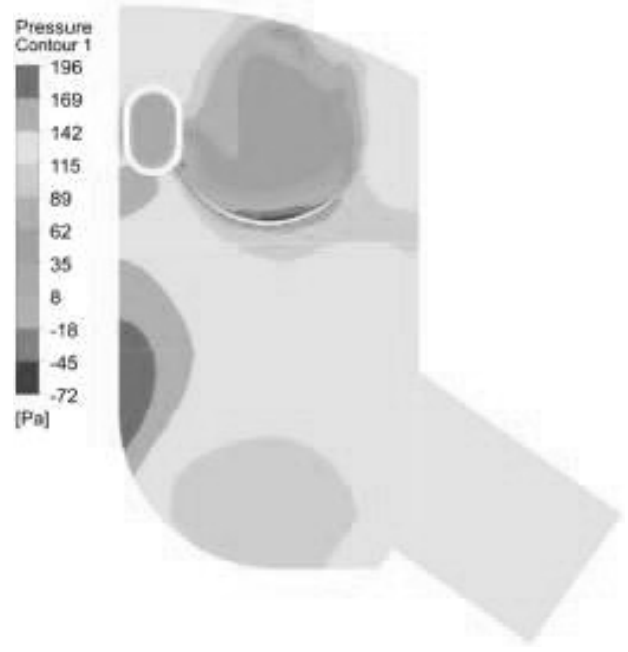

(c) Pressure contour in cross section of middle of air cleaner

Figure 5. Velocity and pressure in air cleaner. 


\section{ANALYSIS OF AIR CLEANER WITH BUILT-IN RESONATOR}

\section{Design of Air Cleaner with Built-In Resonator}

As shown in Figure 6, a wall was designed on the lower part of the air cleaner where the internal flow is least affected, to act as the cavity for the resonator. The upper part and the filter part were the same as those of the conventional model. And in line with the intention to avoid influencing the flow path of the conventional air cleaner, the neck of the resonator was designed to face the inside of the cavity and was located on the right part where there is the least amount of flow. The resonator was designed based on Equation 1, while $160 \mathrm{~Hz}$ was set as the target through additional analysis for precise correction. Other design parameter is shown in Table 3.

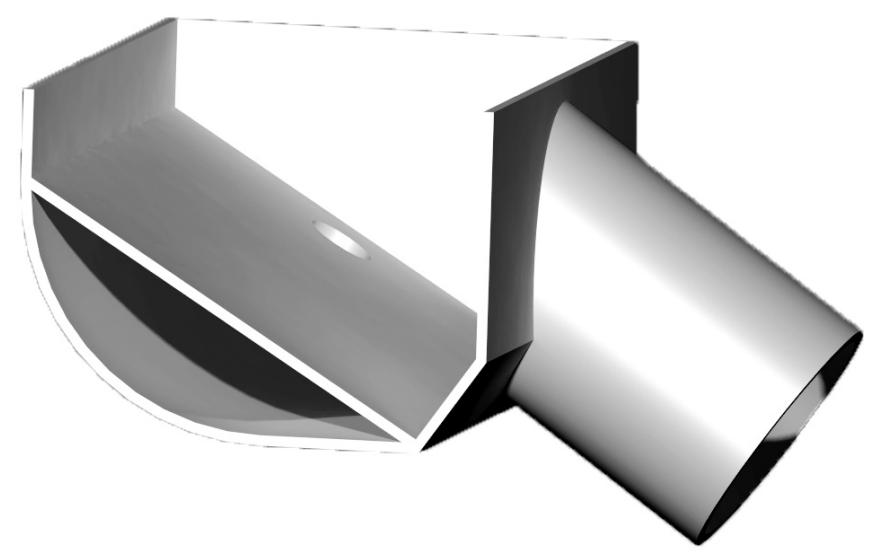

Figure 6. Air cleaner with built-in resonator.

Table 3. Parameter of built-in resonator.

\begin{tabular}{|c|c|}
\hline Name & Value \\
\hline Cavity $\left(V_{c}\right)$ & $567.7 \mathrm{~cm}^{3}$ \\
\hline Length of neck $\left(l_{n}\right)$ & $20 \mathrm{~mm}$ \\
\hline Diameter of neck & $14.4 \mathrm{~mm}$ \\
\hline Cross section or area of neck $\left(A_{n}\right)$ & $162.86 \mathrm{~mm}^{2}$ \\
\hline
\end{tabular}

Table 4. Transmission loss analysis condition.

\begin{tabular}{|c|c|}
\hline Condition & Value \\
\hline Mass density & $1.2047 \mathrm{~kg} / \mathrm{m}^{3}$ \\
\hline Sound speed & $343.24 \mathrm{~m} / \mathrm{s}$ \\
\hline Dynamic viscosity & $1.821 \times 10^{-5} \mathrm{~Pa} \cdot \mathrm{s}$ \\
\hline Bulk viscosity & $0.718 \times 10^{-5} \mathrm{~Pa} \cdot \mathrm{s}$ \\
\hline Thermal conductivity & $0.0256 \mathrm{~W} / \mathrm{m} \cdot \mathrm{s}$ \\
\hline Specific heat $\mathrm{c}_{\mathrm{p}}$ & $1.006 \mathrm{~J} / \mathrm{kg} \cdot \mathrm{K}$ \\
\hline Specific heat $\mathrm{c}_{\mathrm{v}}$ & $0.718 \mathrm{~J} / \mathrm{kg} \cdot \mathrm{K}$ \\
\hline
\end{tabular}




\section{Noise Reduction Characteristics of Air cleaner with Built-In Resonator}

After designing the resonator, we analyzed the noise reduction effect using transmission loss method. Boundary condition used for the transmission loss analysis are in Table 4 , and property of table 4 is the air when the temperature was assumed at $20^{\circ} \mathrm{C}$.

To compare with the conventional air cleaner, analysis was conducted on the $1 \sim 600 \mathrm{~Hz}$ frequency band. Figure 7 shows the TL graph in the air cleaner, where both air cleaners showed reduction effects of $55 \mathrm{~dB}$ at $500 \mathrm{~Hz}$ due to the existing 1/4 wavelength pipe. Also, in the air cleaner with the built-in resonator designed based on Table 2, it was analyzing that in addition to the reduction effect in the $500 \mathrm{~Hz}$ band due to the $1 / 4$ wavelength pipe, there were reduction effects of $61 \mathrm{~dB}$ at the target frequency of $160 \mathrm{~Hz}$, which was the target of the new resonator.

Through this analysis, it was concluded that the air cleaner with built-in resonator acts the role of the conventional air cleaner, as well as a resonator.

Table 5. Pressure drop of each model of air cleaner.

\begin{tabular}{|c|c|}
\hline Model & Pressure Drop \\
\hline Conventional air cleaner & $133.1 \mathrm{~Pa}$ \\
\hline Air cleaner with resonator & $133.5 \mathrm{~Pa}$ \\
\hline
\end{tabular}

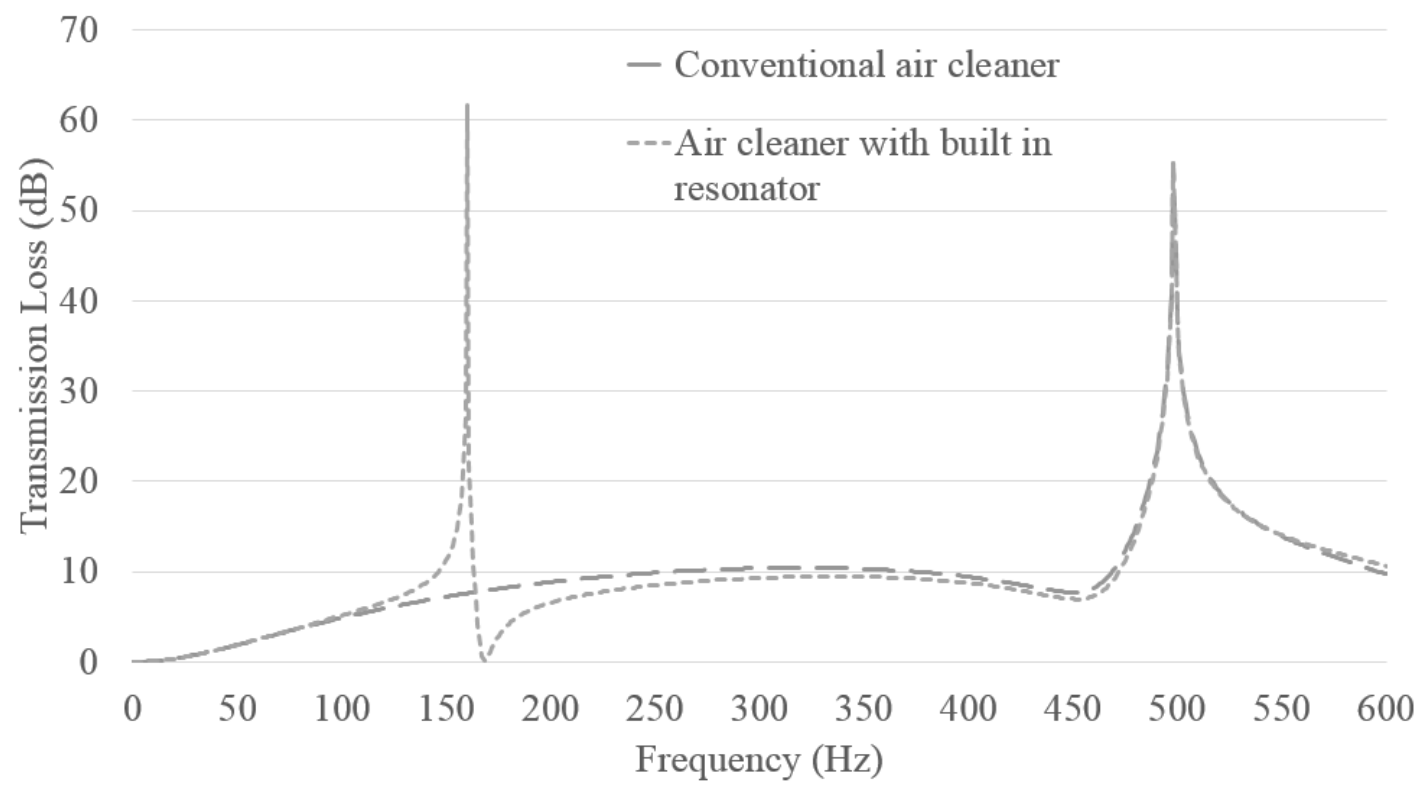

Figure 7. Graph of transmission loss.

\section{Fluid Analysis of Air cleaner with Built-In Resonator}

Fluid analysis was conducted to analyze the effect on flow caused by the resonator added to the air cleaner with built-in resonator. First, the part where the vortex formed in the lower part as shown in Figure 5 was changed to the state shown in (a) and (b) of Figure 8, where such space was eliminated to allow the flow to collide with the wall opposite of the entry part, increasing the flow directly sucked into the diffuser along the wall. Also, as more air collided directly with the wall, pressure was increased along the wall as shown in (c) of Figure 8, and as air moved along the wall, the pressure showed in the conventional air cleaner was drastically reduced. 
Also, Table 3 shows the pressure drop, which is a criterion that can assess the performance of the air intake parts. Both types of air cleaners showed similar results of pressure drop of approximately 133Pa, showing that there were no noticeable performance reductions caused by the resonator.

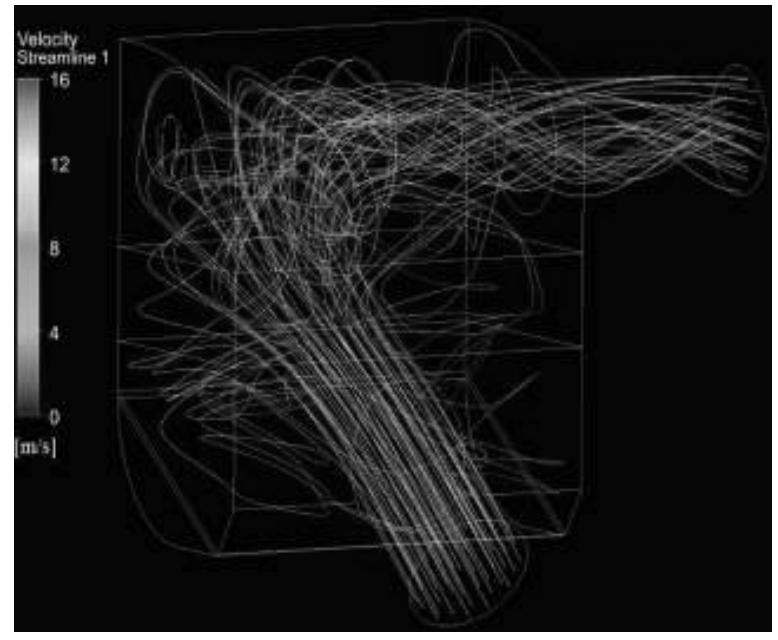

(a) Streamline in air cleaner

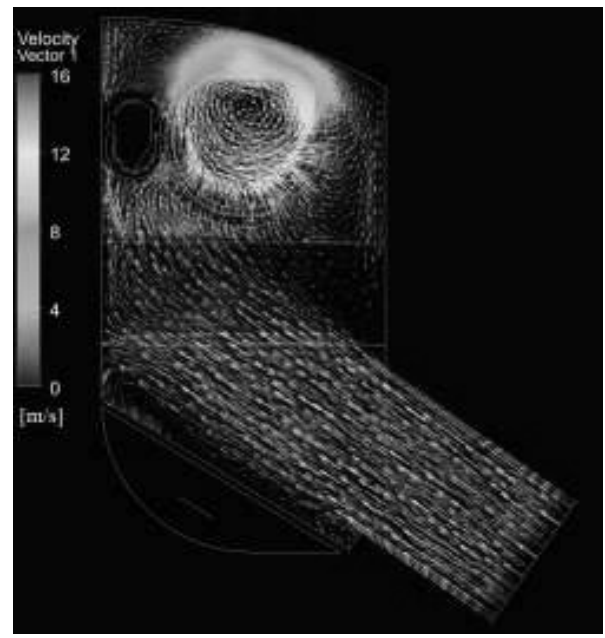

(b) Vector in cross section of air cleaner

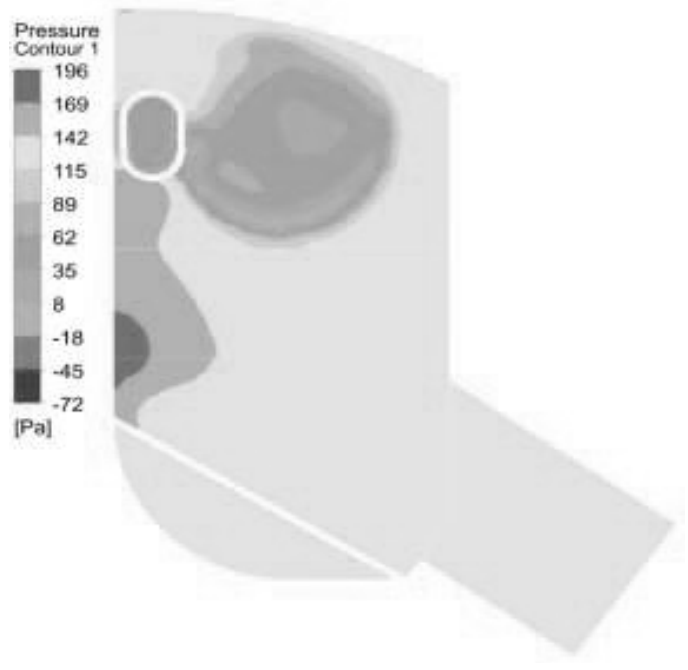

(c) Pressure contour in cross section of middle of air cleaner

Figure 8. Velocity and pressure in air cleaner with built-in resonator.

\section{CONCLUSION}

This study proposed a new type of air cleaner with built-in resonator in order to improve the efficiency of the engine room space in the air cleaner of the intake system. The following results are obtained through the finite element analysis.

1) The flow of the air cleaner with the built-in resonator is more likely to flow along the wall surface than the conventional air cleaner due to the new wall surface, and the lowering of the pressure at the bottom of the diffuser is eliminated. 
2. By attaching the resonator in the lower part of the air cleaner, a noise reduction effect of $61 \mathrm{~dB}$ at $160 \mathrm{~Hz}$ was achieved, and there was no noticeable pressure drop caused by the resonator as compared to the conventional air cleaner.

3. The air cleaner with built-in resonator has a purifying effect using the filter, which does not affect the flow while simultaneously bringing the noise reduction effect as a resonator, thus increasing space efficiency within the engine room.

\section{ACKNOWLEDGMENT}

This research was supported by The Leading Human Resource Training Program of Regional Neo Industry through the National Research Foundation of Korea (NRF) funded by the Ministry of Science, ICT and Future Planning (NRF2016H1D5A1909917).

\& This work was supported by the research grant of the Kongju National University in 2021.

\section{REFERENCES}

A.S. Phulpagar and N.S. Gohel, 2015. CFD Analysis of Air Intake System, International Journal on Theoretical and Applied Research in Mechanical Engineering, 2015, Vol. 4, No. 2, pp. 45-48.

B.M. Yang, H.M. Cho, 2016. "Flow Analysis of Air Intake Duct for Noise Reduction in Automobile", Contemporary Engineering Sciences, Vol.9, No.20, pp. 989-995.

D.T. Hong, B.K. Son Y.H. Kim, T.B. Kim and J.M. Kang, 2010. A Study on the Development of the Perforated Resonator of Air Induction System for Small Vehicle, The Korean Society of Automotive Engineers, Annual autumn seminar, pp. 2365-2369.

H.Y. Song and D.H. Lee, 2008. A Study in the Acoustic Absorption Performance of a Helmholtz Resonator, Korean Society for Noise and Vibration Engineering, Vol. 18, No. 1, pp. 71-79.

J. Lee, 2009. Effect of Leakage on the Noise Reduction Characteristics of Helmholtz Resonator, Korean Society for Noise and Vibration Engineering, Vol. 19, No. 6, pp. 634-640.

J.E. Kim, 2015. Helmholtz resonator, Korean Society for Noise and Vibration Engineering, Vol. 25, No. 6, pp. 24-28.

J.G. Ih, H.J. Kim, S.H. Lee, K. Shinoda, 2009. Prediction of intake noise of an automotive engine in run-up condition", Applied Acoustics, Vol.70, pp. 347-355.

M.C. Brands, 1979. Helmholtz Tuned Induction System for Turbocharged Diesel Engine in 1979 Automotive Engineering Congress and Exposition.

M.S. Kim, S.D. Kang and J.W. Lee, 2006. Reduction intake noise by resonator tuning, The Korean Society of Automotive Engineers, Annual autumn seminar, pp. 1767-1772.

S. Allam, 2015. Low Noise Intake System Development for Turbocharged I.C. Engines Using Compact High Frequency Side Branch Resonators, Advances in Powertrains and Automotive, Vol.1, No.1, pp. 12-23.

S. Kitahara, H. Takao, T. Hashimoto, S. Hatano, 2005. Improvement of Car Interior Noise by Utilizing a Porous Intake Duct: Treatment Effect on an Intake System, SAE Noise and Vibration Conference and Exhibition.

S.H. Kim, K.J. Lee and W.S. Lee, 2014. Research for Reduction Frequency characteristic of perforated resonator about Length of Extended Neck, The Korean Society of Propulsion Engineer, Annual spring seminar, pp. 342-347.

S.K. Tang, 2005. On the Helmholtz Resonators with Tapered Necks, Journal of Sound and Vibration, Vol. 279, pp.1085-1096.

Tetsushi Suzuki \& Fumihiko Kayaba, 1990. The analysis and mechanism of engine Intake Rumbling Noise, SAE 901755

U. Patil and D. Rahane, 2019. Design, Simulation \& Optimization of an Air Intake System to Reduce Induction Noise," in Symposium on International Automotive Technology.

V. Vaidya, P.P. Hujare, 2014. Effect of Resonator on Transmission Loss and Sound Pressure Level of an Air Intake System, International Journal of Engineering and Advanced Technology, Vol.3, No.3, pp. 349-351

Vishal Vaidya, P.P. Hujare, 2014. "Effect of Resonator on Transmission Loss and Sound Pressure Level of an Air Intake System, Vol.3, No.3, pp. 349-351.

Y. EInemr, 2011. Acoustic Modeling and Testing of exhaust and intake system components, KTH Royal Institute of Technology, p. 94. 